\section{LA HOMOFOBIA EN LA EDUCACION FISICA ESCOLAR: UNA REVISIÓN SISTEMÁTICA}

\author{
A HOMOFOBIA EM EDUCAÇÃO FÍSICA NA ESCOLA: UMA REVISÃO

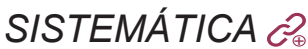

HOMOPHOBIA IN SCHOOL PHYSICAL EDUCATION: A SYSTEMATIC REVIEW CP

doi' $\mathrm{https://doi.org/10.22456/1982-8918.104750}$

\footnotetext{
DD Angélica María Sáenz-Macana* <angie8866@gmail.com>

José Devís-Devís** <jose.devis@uv.es>
}

\begin{abstract}
*Secretaria de Educación del Distrito, Bogotá, Colombia y Universitat de València. Valencia, España.

**Universitat de València. Valencia, España.
\end{abstract}

Resumen: La Educación Física (EF) es una de las asignaturas escolares que más promueve un ambiente hostil y marginal para las personas homosexuales. Por ello, este trabajo tiene como objetivo analizar los artículos empíricos sobre las creencias, las actitudes y los comportamientos homofóbicos presentes en la EF escolar mediante una revisión sistemática. Además de varios aspectos bibliométricos de interés, emergieron cuatro grandes temas derivados del análisis: 1) la heteronormatividad y el heterosexismo imperante; 2) la existencia de homopositividad; 3) la homonegatividad atenuada; y 4) las propuestas para la acción. Estos temas se discuten en detalle y se concluye que pese a observarse mejoras, la homofobia todavía está muy extendida. Además, resulta necesario investigar la homofobia con muestras variadas y con diversidad de métodos, porque todavía queda mucho por conocer, ya sea sobre variables o en contextos personales, sociales y ambientales diferentes.

Palabras clave: Homofobia. Normas de género. Revisión sistemática. Educación Física.
Recebido em: 25-06-2020 Aprovado em: 11-08-2020 Publicado em: 19-09-2020

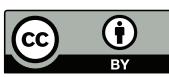

Este é um artigo publicado sob a licença Creative Commons Atribuição 4.0 Internacional (CC BY 4.0). elSSN: $1982-8918$ 


\section{INTRODUCCIÓN}

El término homofobia fue creado por George Weinberg en la década de 1970 para referirse a los sentimientos negativos que provocaba la homosexualidad (HILL; WILLOUGHBY, 2005; LONGARAY; RIBEIRO; SILVA, 2011; MADŽAREVIC, 2016), como el temor de estar con un homosexual en un espacio cerrado (COSTA; BANDEIRA; NARDI, 2013; HEREK; MCLEMORE, 2013; MORENO et al., 2015), y a la actitud negativa e irracional hacia las personas homosexuales (FRAÏSSÉ; BARRIENTOS, 2016; MORROW; GILL, 2003; MOSCOSO; PIEDRA, 2019). Posteriormente, algunos autores propusieron reemplazarlo por el término homonegatividad y homonegativismo (HUDSON; RICKETTS, 1980), prejuicio sexual (HEREK, 1984), homoprejuicio (LOGAN, 1996) u homodiscriminación (TENA, 2012) porque el sufijo fobia no se ajustaba al contenido conceptual que recoge la literatura al respecto.

Sin embargo, la homofobia sigue siendo reconocida como una palabra clave en este tema hasta el punto de entenderse como un término paraguas bajo el que se ampara al conjunto de actitudes negativas que denotan prejuicios sociales e institucionales hacia los hombres gays y las mujeres lesbianas (HEREK, 2004; 2013). Algunos autores incluso han ampliado recientemente el término a las actitudes negativas hacia el amplio grupo de las personas LGBT+, quienes no se ajustan a los roles y estereotipos tradicionalmente establecidos (RAMÍREZ-DÍAZ; CABEZARUIZ, 2020; SILVA; JAEGER; VALDIVIA-MORAL, 2018). La homofobia no puede entenderse solamente como los rechazos de unas individualidades hacia las personas homosexuales sino un conjunto de creencias, actitudes y prácticas que se construyen social y culturalmente (LENSKYJ, 1991; PICHARDO, 2012). La homofobia constituye una estrategia de control social que permite la discriminación y subalternidad de quienes se apartan del heterocentrismo o sitúan a la heterosexualidad en el centro de la vida social (BORRILLO, 2001). Es un fenómeno muy complejo que implica a diversos procesos sociales y se alinea con los valores masculinos y el heterosexismo como los valores supremos o más importantes para el orden, la estabilidad y el funcionamiento globalizado de la sociedad (CONNELL; PEARSE, 2018).

El heterosexismo es uno de los pilares sobre los que se apoya la homofobia para su extensión social. Sears y Williams (1997) lo definieron como "una creencia en la superioridad de los heterosexuales y la heterosexualidad evidenciada por la exclusión, omisión o diseño de políticas, procedimientos, eventos o actividades de personas no heterosexuales" (traducción nuestra) (p. 16). Se afirma, entonces, que el heterosexismo promueve una actitud hostil, violenta y discriminatoria hacia la homosexualidad. Se convierte en un rechazo silencioso e institucionalizado hacia las personas identificadas como homosexuales, limitando su acceso a derechos, espacios, reconocimiento, prestigio o poder (PICHARDO, 2009).

La heterosexualidad, cuando se asocia con los valores masculinos dominantes, acaba privilegiando la masculinidad sobre la feminidad y la homosexualidad. Por ello, Connell y Pearse (2018) señalan que se han promovido actitudes y comportamientos misóginos y homofóbicos que mantienen una estructura jerárquica en la sociedad y la masculinidad hegemónica, a través del funcionamiento de las principales 
instituciones sociales como el estado, la empresa, los medios de comunicación y la religión. Por ende, ha dado lugar a prejuicios construidos históricamente y con fuerte enraizamiento afectivo que han acabado por constituirse en barreras culturales hacia los homosexuales (MADUREIRA; BRANCO, 2015).

Por otro lado, el discurso heteronormativo dominante o hegemónico se perpetúa y reproduce también en la escuela como espacio vital de socialización para las jóvenes generaciones (CORNEJO, 2014; FERFORLJA, 2010; JOY; LARSSON, 2019; LANDI, 2018; LENSKYJ, 1991; MADUREIRA; BARRETO, 2018; PRADO; RIBEIRO, 2015). Por consiguiente, la escuela, tal como describe Prado y Ribeiro (2015), se torna en un espacio de expansión de nuestros prejuicios y discriminaciones, en ocasiones, la escolarización puede convertirse en una experiencia torturante para los estudiantes que no se enmarcan en los estándares de género y sexualidad hegemónicos. Es así como puede convertirse en un ambiente de aprendizaje de la discriminación y no como en un ambiente inclusivo.

Además, la educación física (EF) se considera como una de las asignaturas escolares que más contribuye al refuerzo de la ideología sexista (AYVAZO; SUTHERLAND, 2009; PEREIRA et al., 2015; PRADO; RIBEIRO, 2016). Esta asignatura respalda los estereotipos masculinos y femeninos tradicionales durante la práctica deportiva debido a la predominante separación sexual en el desarrollo de muchas actividades (CLARKE, 2012; DEVÍS-DEVÍS et al., 2018; LARSSON; REDELIUS; FAGRELL, 2011; NICOLINO; PARAISO, 2018; SCHARAGRODSKY, 2003). En este entorno se experimentan regularmente lenguajes y comportamientos homofóbicos y heterosexistas (CUNHA JÚNIOR; MELO, 1996; MORROW; GILL, 2003) que promueven un ambiente hostil para los homosexuales (HEMPHILL; SYMONS, 2009; MCCAUGHTRY et al., 2005; PIEDRA et al., 2014). Por ello, resulta un espacio privilegiado para valorar los estereotipos, roles y creencias de género presentes en las aulas y en las prácticas docentes.

La discriminación y la inseguridad también la experimentan jóvenes LGBTQ+ en otros contextos de práctica física y deportiva (GREENSPAN; GRIFFITH; WATSON, 2019). Incluso las profesoras lesbianas y los profesores gay de EF también experimentan la intimidación y la vulnerabilidad (CLARKE, 1998; DEVÍS; FUENTES; SPARKES, 2005; GRIFFIN, 1998; SQUIRES; SPARKES, 1996; SYKES; GOLSTEIN, 2004). No obstante, es pertinente señalar que algunos estudios recientes revelan una cierta disminución de los niveles de homofobia y mayor tolerancia hacia los homosexuales en el ámbito deportivo (ANDERSON; BULLINGMAN, 2015; PIEDRA; GARCÍA-PÉREZ; CHANNON, 2017). Es por ello que resulta necesario incrementar la investigación en la EF escolar para conocer el alcance e impacto de la homofobia en esta asignatura.

Un primer paso consiste en conocer el estado de la cuestión sobre esta temática mediante una revisión sistemática que concentre el conocimiento existente hasta este momento. De esta manera, pueden identificarse los avances, los saberes consolidados y las lagunas o terrenos inexplorados que orienten el futuro desarrollo de la investigación sobre la homofobia y la EF. Por ello, el objetivo de este artículo consiste en analizar los artículos empíricos sobre las creencias, las actitudes y los 
comportamientos homofóbicos presentes en la EF escolar, tanto si se han realizado con muestras de alumnado, profesorado o adultos que recuerdan su escolarización, desde el año 2007 que termina la última revisión (AYVAZO; SUTHERLAND, 2009) hasta marzo de 2020.

\section{METODOLOGIA}

Las revisiones sistemáticas son las formas más comunes de adquirir conocimiento amplio y condensado sobre determinados temas de investigación (GRANT; BOOTH, 2009) que siguen un proceso metodológico riguroso (ZAWACKIRITCHER et al., 2020). Por tanto, no se considera una mera revisión narrativa de la literatura publicada sino un tipo de investigación científica que parte de un propósito, sigue una metodología específica y alcanza una serie de resultados sobre la temática de estudio (FERREIRA; URRUTIA; ALONSO-COELLO, 2011). Recientemente, Landi et al. (2020) han publicado una revisión narrativa, más general que la revisión sistemática, sobre temática LGBTQ en educación física que no está específicamente orientada a la homofobia.

Para la identificación de los artículos pertinentes al propósito de estudio se realizó una búsqueda en las siguientes bases de datos internacionales: Web of Science (WOS), Pubmed, Scopus, Sportdiscus, ERIC, y Dialnet. La estrategia de búsqueda para operadores booleanos se concreta en esta fórmula: ((Homophobi* OR Homonegativ* OR Homoprejudi*) AND ('Physical Education') AND (Student OR Teacher)). Para la búsqueda en Dialnet también se utilizaron los respectivos equivalentes en español. Además, se colocó el asterisco* para que alcanzara a todos los sufijos posibles. Los términos incluidos en la fórmula son los más comunes en la literatura sobre el tema, tal y como se indica en el marco teórico presentado en la introducción de este trabajo.

Los criterios de inclusión utilizados para identificar los documentos relevantes para esta revisión son: 1) artículos empíricos, ya sean de metodología cuantitativa y/o cualitativa; 2) que deben utilizar muestras de alumnado, profesorado de EF escolar, y/o adultos que recuerden experiencias en EF escolar; 3) que se encuentren escritos en inglés, español o portugués; y 4) estén publicados entre enero de 2007 y marzo de 2020. Los criterios de exclusión fueron: 1) documentos que no son artículos científicos como capítulos de libro, revisiones o documentos de carácter teórico; y 2) documentos que no se dedican a la homofobia en EF escolar como puede ser el deporte competitivo, el análisis de prácticas pedagógicas de EF y a las políticas educativas inclusivas.

\section{RESULTADOS Y DISCUSIÓN}

\subsection{ASPECTOS BIBLIOMÉTRICOS}

La aplicación de la fórmula de búsqueda arrojó 66 artículos procedentes de las seis bases de datos consultadas, distribuyéndose de la forma siguiente: 22 procedentes de WOS, 3 de Pubmed, 15 de Scopus, 16 de Sportdiscus, 6 de ERIC 
y 4 de Dialnet. Además, se incluyeron dos artículos obtenidos de las referencias observadas en artículos revisados. En el diagrama de flujo (Figura 1) se especifica el proceso de selección de los artículos hasta quedar los 10 que se someten a un análisis en profundidad.

Figura 1 - Diagrama de Flujo de la selección de estudios

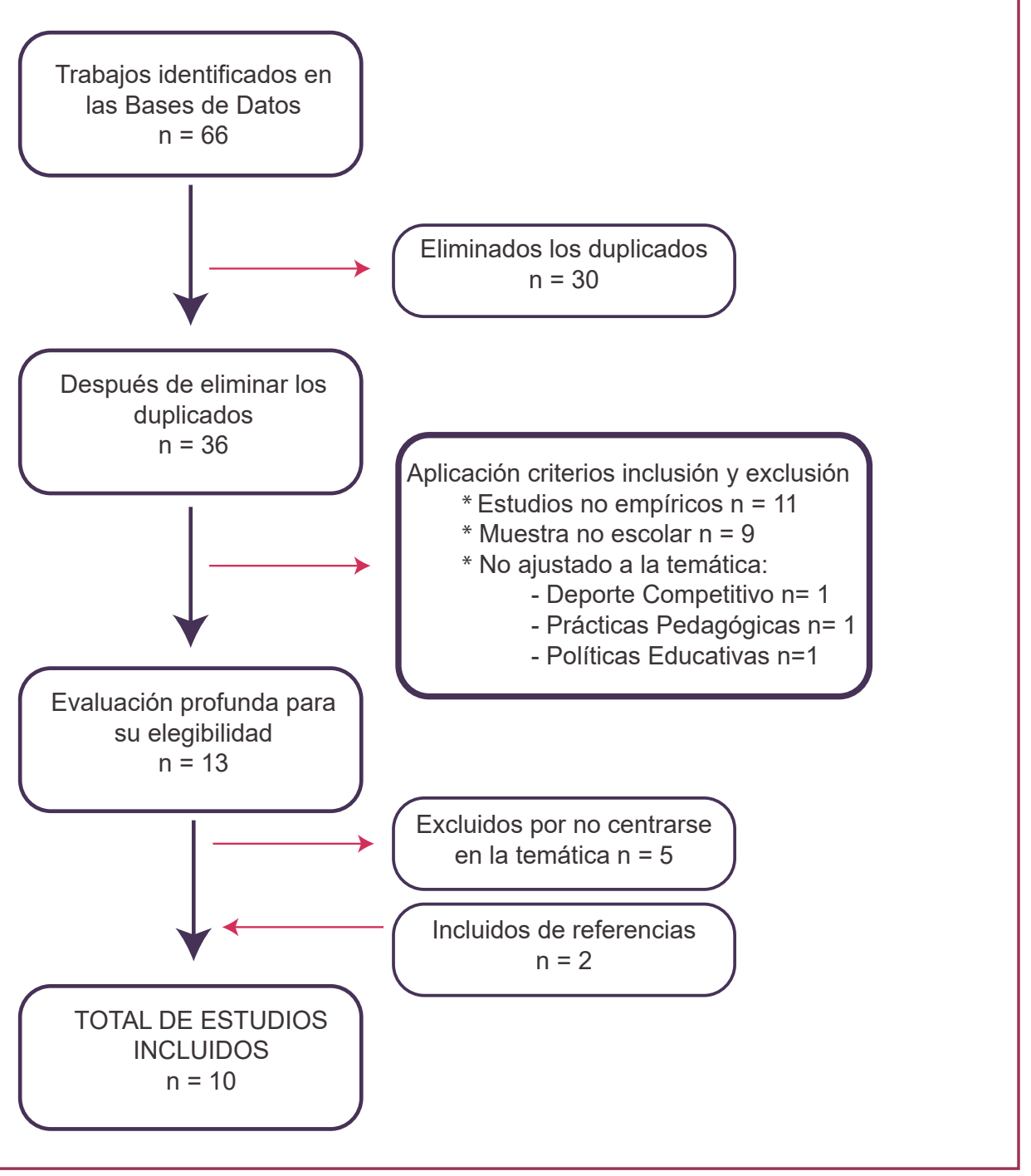

Fuente: Elaborado por autores

En la Tabla 1 se observa que desde 2007 existe un creciente número de publicaciones proceden de países en los que existe legislación a favor de la igualdad sexual como, por ejemplo, el matrimonio homosexual 
Tabla 1 - Principales Estudios Identificados

\begin{tabular}{|c|c|c|c|c|}
\hline Autoría (Año) & País & Participantes & Metodología Utilizada & Principales Hallazgos \\
\hline Anderson (2012) & UK & $\begin{array}{l}\text { Aprox. } 100 \text { Escolares en la } \\
\text { etnografía } \\
17 \text { Escolares en las entrevistas }\end{array}$ & $\begin{array}{l}\text { Etnografía de } 3 \text { meses } \\
\text { Entrevistas en } \\
\text { profundidad }\end{array}$ & $\begin{array}{l}\text { Se evidencia ausencia de discurso homofóbico, reducción de violencia, } \\
\text { ausencia de hiper-masculinidad, mayor aceptación homosexual y apoyo } \\
\text { emocional por parte de pares }\end{array}$ \\
\hline $\begin{array}{l}\text { Beserra, Brito, } \\
\text { Ribeiro (2019) }\end{array}$ & BRA & $\begin{array}{l}4 \text { Docentes de EF } \\
40 \text { Escolares }\end{array}$ & $\begin{array}{l}\text { Cuestionarios propios } \\
\text { Entrevistas-diálogo }\end{array}$ & $\begin{array}{l}\text { Se presencian agresiones verbales hacia los homosexuales, el } \\
\text { profesorado intenta actuar frente a la homofobia, pero no es suficiente } \\
\text { para reducirla en la escuela }\end{array}$ \\
\hline Gill et al. (2010) & USA & $\begin{array}{l}\text { Fase 1: } 149 \text { universitarios en } \\
\text { Ciencias del Deporte } \\
\text { Fase 2: } 22 \text { Universitarios en } \\
\text { Ciencias del Deporte } \\
27 \text { Universitarios del Campus } \\
\text { Pride Group }\end{array}$ & $\begin{array}{l}\text { National School Climate } \\
\text { Survey } \\
\text { Physical Activity Climate } \\
\text { Measure }\end{array}$ & $\begin{array}{l}\text { Se percibe a la EF como un entorno hostil para el alumnado LGBT, hay } \\
\text { exclusión de grupos minoritarios en actividades físicas escolares, se } \\
\text { reconocen los insultos homofóbicos y acoso continuo a los LGBT }\end{array}$ \\
\hline $\begin{array}{l}\text { Piedra et al. } \\
\text { (2016) }\end{array}$ & ESP & 170 Docentes de EF & $\begin{array}{l}\text { Cuestionario Percepción } \\
\text { de la Homofobia y } \\
\text { Heterosexismo en EF }\end{array}$ & $\begin{array}{l}\text { El comportamiento heterosexista y homofóbico es evidente en las } \\
\text { lecciones de EF y el profesorado es consciente de esto, los estudiantes } \\
\text { usan el lenguaje para discriminar a gays y lesbianas }\end{array}$ \\
\hline $\begin{array}{l}\text { Piedra et al. } \\
\text { (2013) }\end{array}$ & ESP & $\begin{array}{l}245 \text { Universitarios de la } \\
\text { Facultad de Ciencias de la } \\
\text { Educación }\end{array}$ & $\begin{array}{l}\text { Cuestionario Percepción } \\
\text { de la Homofobia y } \\
\text { Heterosexismo en EF }\end{array}$ & $\begin{array}{l}\text { Generación de conductas heterosexistas, alto porcentaje de estudiantes } \\
\text { que han sido testigos o han experimentado comportamientos homofóbicos, } \\
\text { falta de concienciación al respecto entre el profesorado }\end{array}$ \\
\hline Prado (2017) & BRA & 6 Adultos & $\begin{array}{l}\text { Entrevistas } \\
\text { semiestructuradas }\end{array}$ & $\begin{array}{l}\text { Se infiere que el estudiantado que no encaja en los modelos hegemónicos } \\
\text { son blanco de las demarcaciones despectivas, aquellos que chicos que } \\
\text { son más femeninos son objetos de burla y acoso }\end{array}$ \\
\hline $\begin{array}{l}\text { Prado, Ribeiro } \\
(2016)\end{array}$ & BRA & 6 Adultos & $\begin{array}{l}\text { Entrevistas } \\
\text { semiestructuradas }\end{array}$ & $\begin{array}{l}\text { Se evidencia que las practicas corporales y deportivas enmarcadas en } \\
\text { discurso binario, promueven la estigmatización y discriminación a las } \\
\text { sexualidades no heterosexuales en EF }\end{array}$ \\
\hline $\begin{array}{l}\text { Silva, Jaeger, } \\
\text { Valdivia-Moral } \\
(2018)\end{array}$ & PRT & 223 Universitarios de EF & $\begin{array}{l}\text { Cuestionario Percepción } \\
\text { de la Homofobia y } \\
\text { Heterosexismo en EF }\end{array}$ & $\begin{array}{l}\text { Los comportamientos heterosexistas se observaron más frecuentemente } \\
\text { que los homofóbicos en las clases, las mujeres son más sensibles a su } \\
\text { percepción }\end{array}$ \\
\hline
\end{tabular}

Fuente: Elaborada por autores

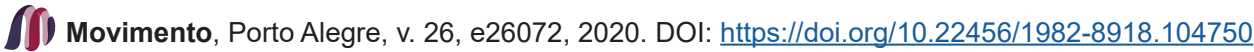


Específicamente, España y Brasil cuenta con tres artículos publicados, le sigue Reino Unido con dos artículos, y Portugal y Estados Unidos con un solo artículo. No obstante, en la mayoría de ellos todavía no existe una legislación educativa que reconozca explícitamente los contenidos de educación sexual diversa e igualdad por razón de orientación sexual en el currículo escolar. Solamente el Reino Unido incluye contenidos sobre orientación sexual e identidades de género en el currículum de secundaria y sobre tipos de familia, incluida la LGBT, en el currículum de primaria (RELATIONSHIPS..., 2019).

Por otro lado, es interesante observar que cuatro artículos utilizaron metodología cuantitativa, cinco cualitativa y uno metodología mixta. Los trabajos cuantitativos presentaron un diseño tipo survey mediante el uso de dos cuestionarios. El cuestionario de 'Percepción de la Homofobia y Heterosexismo en EF' (MORROW; GILL, 2003) lo utilizaron en tres artículos y el otro restante usó la 'Encuesta Nacional del Clima Escolar' (KOSCIW; DIAZ, 2006) y una medida del clima de actividad física, desarrollada específicamente para el estudio. Los trabajos cualitativos utilizaron la etnografía y las entrevistas semiestructuradas como estrategias metodológicas, en uno y tres artículos respectivamente. En el trabajo mixto se aplicaron dos cuestionarios elaborados específicamente para el estudio y una entrevista tipo diálogo para profundizar en las respuestas de los cuestionarios.

Las muestras utilizadas en los estudios de esta revisión fueron diversas en tamaño y dependientes del tipo de metodología. Es decir, las muestras pequeñas correspondían a estudios que utilizaban estrategias metodológicas cualitativas que profundizaban y aportaban detalles narrativos, mientras que las grandes muestras correspondían a trabajos cuyas estrategias metodológicas cuantitativas aportaban menos detalles y más datos generalizables. Resulta destacable que sólo dos estudios se realizaron con participantes escolares y los ocho estudios restantes con universitarios y personas adultas que recordaban sus clases de EF escolar. Las muestras de cuatro de estos últimos artículos fueron docentes de EF. Esto refleja la dificultad de realizar investigaciones sobre la homofobia en estudiantes menores de edad, probablemente por razones éticas, de permiso de los padres/madres/tutores, y por la falta de legislación estatal al respecto que permita abordar abiertamente y sin tabúes el tema con los escolares.

Por último, aunque se trata de artículos que proceden de estudios empíricos, la gran mayoría de los participantes no suelen ser personas homosexuales. En los estudios cualitativos solo fueron 15 personas declaradas gays o lesbianas, en los estudios cuantitativos la mayor muestra de no heterosexuales se encuentra en el estudio de Gill et al., (2010) no superando el 12\%. En el estudio mixto no se especifica nada al respecto. En general, se observa una tendencia en los estudios a ocuparse de las percepciones y actitudes de la población general o personas heterosexuales hacia las personas homosexuales en la EF escolar.

\subsection{HETERONORMATIVIDAD Y HETEROSEXISMO IMPERANTE}

De los diez estudios analizados, nueve consideran que la heteronormatividad y el heterosexismo siguen reproduciéndose en la escuela y con gran presencia en las 
aulas de EF. Además, afirman que genera marginación y discriminación al alumnado y profesorado que no está en consonancia con los valores heterosexuales considerados tradicionales. El único estudio que se aleja de esa descripción es el de Anderson (2012) porque los adolescentes participantes en su etnografía señalan gran aceptación e inclusión de la homosexualidad en la escuela. Los valores heterosexistas indican que el aspecto físico, la apariencia corporal y las habilidades físicas privilegian una masculinidad hegemónica (hombre, heterosexual, blanco y atlético). Esto favorece la aparición de creencias erróneas, tales como que los homosexuales son inferiores, débiles atléticamente o son contrarios al ideal deportivo (PRADO, 2017).

Curiosamente, estas etiquetas homofóbicas han sido atribuidas anteriormente a las mujeres. En la actualidad, como indican diversos autores (GILL et al., 2010; PIEDRA et al., 2016; PRADO; RIBEIRO, 2016), los hombres que no son atléticos $y$ heterosexuales son excluidos y las mujeres que son fuertes y atléticas son consideradas lesbianas y no femeninas. Por consiguiente, toda persona que rompa el binarismo asociado a la coherencia sexo/género y que no corresponda con una atracción heterosexual puede verse ridiculizada y perjudicada por conductas e insultos homofóbicos, tanto en España (PIEDRA et al., 2013), en Portugal (SILVA; JAEGER; VALDIVIA-MORAL, 2018), en Reino Unido (EDWARDS; BROWN; SMITH, 2016), en Estados Unidos (GILL et al., 2010) o en Brasil (PRADO, 2017).

Los estudios realizados con profesorado de EF también señalan el predominio de una visión binaria de género en la enseñanza de la EF porque asumen la competición y las prácticas deportivas divididas por sexo, es decir, según si son prácticas de chicas o de chicos (PIEDRA; RAMÍREZ-MACÍAS; LATORRE, 2014; PRADO, 2017; PRADO; RIBEIRO, 2016). Incluso adaptan los deportes y las pruebas atléticas a las chicas porque son consideradas más débiles que los chicos, prueba del estatus superior que asignan a la masculinidad y a sus valores asociados (virilidad, heterosexualidad, sexismo y misoginia) (PRADO, 2017). Por ende, se evidencia que el modelo establecido por la sociedad $y$, particularmente, en las clases de EF parece corresponderse con la fuerza, competitividad, agresividad, virilidad y heterosexualidad (PIEDRA; RAMÍREZ-MACÍAS; LATORRE, 2014; SILVA; JAEGER; VALDIVIA-MORAL, 2018). Por contra, aquellos estudiantes que no cumplan con este modelo pueden ser víctimas de acoso (GILL et al., 2010) y vivenciar sentimientos de tristeza, vergüenza e impotencia (PRADO; RIBEIRO, 2016).

Asimismo, como enuncia Silva, Jaeger y Valdivia-Moral (2018), el conjunto de prácticas pedagógicas escolares en EF está influenciado por los mismos estereotipos y restricciones heterosexistas que en el deporte, tal y como también recogen otros autores (GILL et al., 2006; GREENSPAN; GRIFFITH; WATSON, 2019; JAEGER, et al., 2019; RAMÍREZ-DÍAZ; CABEZA-RUIZ, 2020).

\subsection{EXISTENCIA DE HOMOPOSITIVIDAD}

En el apartado anterior se aportan evidencias de la heteronormatividad y el heterosexismo existente en la EF que proceden de los artículos objeto de análisis de esta revisión. Sin embargo, también se exhiben algunas actitudes y/o comportamientos positivos hacia las personas homosexuales. 
Resulta importante mencionar que el trabajo de Anderson (2012) es el único estudio que señala una disminución de la homofobia entre estudiantes de secundaria postobligatoria del Reino Unido. En particular, los participantes manifiestan que la homofobia es considerada como un signo de inmadurez o infantilismo, además de considerar que ser homofóbico es tan grave como ser racista. Este trabajo da a entender que después de la pubertad parece existir un trato más respetuoso con la homosexualidad y las personas homosexuales. Además, en esta etnografía también se da cuenta de una reducción de la homohisteria entre los adolescentes o la falta de temor por la demostración de afecto entre pares. Es decir, tanto las personas heterosexuales como homosexuales expresan abiertamente su amor, se abrazan en público, exhiben su cariño de manera verbal y en redes sociales, y se apoyan entre sí (ANDERSON, 2012). Esta disminución de la homohisteria también parece observarse en el contexto español de la EF escolar a tenor de la aceptación y menor hostilidad hacia la homosexualidad entre escolares (PIEDRA; RAMÍREZ-MACÍAS; LATORRE, 2014). Asimismo, en el estudio de Beserra, Brito y Ribeiro (2019), la muestra de adolescentes también presenta ciertas actitudes positivas de tolerancia hacia la homosexualidad.

Según lo expuesto anteriormente, parece ser que se están forjando nuevas masculinidades y feminidades al ser aceptadas y toleradas otras maneras de ser, sentir y querer, lo que podría favorecer ambientes de clase más inclusivos. Un adolescente del estudio de Anderson (2012) señala que la popularidad entre ellos ya no consiste en ser considerado un hipermacho o agresivo, sino en tener seguridad personal, mostrarse más emocional, apoyar a los compañeros y tener empatía. El alumnado adolescente, en general, consideran que practican deporte para generar vínculos sociales y no para mantener su heteromasculinidad. Incluso parecen estar cambiando las representaciones de la feminidad, ya que las chicas del estudio brasileño de Prado (2017) que demostraban habilidades físicas, motoras y un sentido de competitividad alto eran aceptadas y solicitadas a la hora de formar los equipos deportivos.

En varios de los estudios de esta revisión se indica que la mayoría de los participantes no fueron objeto de comentarios homofóbicos ni heterosexistas por parte de otros estudiantes o profesores (PIEDRA et al., 2013; SILVA; JAEGER; VALDIVIAMORAL, 2018) o de sus estudiantes o profesores colegas (PIEDRA et al., 2016). Es posible que esto se deba, a la gran cantidad de participantes heterosexuales en las muestras en comparación con los no heterosexuales. Además, tanto estudiantes como docentes manifiestan una elevada utilización del lenguaje inclusivo durante las clases, lo que indica un gran avance en la EF escolar.

Finalmente, se destaca en el profesorado de EF la preocupación que muestran por hacer frente a la discriminación en sus clases (BESERRA; BRITO; RIBEIRO, 2019; EDWARDS; BROWN; SMITH, 2016; PIEDRA; RAMÍREZ-MACÍAS; LATORRE, 2014; PIEDRA et al., 2016). El colectivo de profesorado parece estar interesado en cambiar la discriminación y desigualdad mediante la creación de espacios seguros, la eliminación de comportamientos homofóbicos y la concienciación de ello entre el alumnado. Así lo señala también el estudio de Gill et al. (2010), ya que los estudiantes 
universitarios afirman que durante su periodo de escolarización algunos docentes se enfrentaban a situaciones discriminatorias en sus clases, aunque no lograsen hacerlas desaparecer del todo.

\subsection{HOMONEGATIVIDAD ATENUADA}

La percepción de los comportamientos heterosexistas y homofóbicos se produce tanto entre el profesorado como entre el alumnado, siendo más frecuentes los primeros comportamientos que los segundos y en mayor número en las relaciones entre estudiantes que entre profesorado y estudiantes (GILL et al., 2010; PIEDRA et al., 2013; PIEDRA et al., 2016; SILVA; JAEGER; VALDIVIA-MORAL, 2018). Esto puede deberse a que los comportamientos heterosexistas se ven como normales y no como una forma de discriminación por estar muy extendidos (PIEDRA et al., 2016). También puede ser porque el profesorado se autocontrola cuando se relaciona con su alumnado, ya que se le considera un profesional con autoridad moral que procura por un ambiente respetuoso en sus clases.

Por otra parte, la mayoría del alumnado y el profesorado encuestado manifiesta que regularmente han sido testigos de comportamientos homonegativos y pocos manifiestan haber sido víctima en dichas situaciones (PIEDRA et al., 2013; PIEDRA et al., 2016; SILVA; JAEGER; VALDIVIA-MORAL, 2018). Esto puede explicarse porque la mayoría de los encuestados son heterosexuales y aquellos que no lo son, tienden a ocultarlo ante los demás. También resulta interesante señalar que las mujeres del estudio de Silva, Jaeger y Valdivia-Moral (2018) perciben con más frecuencia los comentarios homofóbicos. Sin embargo, los hombres los usan más que las mujeres, aunque también parecen ser víctimas frecuentes, tal y como recoge el estudio español de Piedra et al. (2016).

Algunos trabajos revisados señalan que estudiantes de distintos países utilizan muy frecuentemente el uso de palabras hirientes de connotación sexual para ofenderse entre ellos, tales como maricón o bollera en inglés (faggot o dyke) (GILL et al., 2010) o portugués (viadinho o sapatão) (PRADO, 2017). Incluso utilizan bromas, chistes y expresiones jocosas para disimular los insultos ofensivos (PRADO; RIBEIRO, 2016). Es más, los lugares de la escuela donde se escuchan más comentarios homofóbicos de este estilo son los pasillos, los vestuarios y gimnasios o campos de deporte, presentándose menos insultos en las aulas de clase (GILL et al., 2010). Es así como queda en evidencia que el lenguaje peyorativo sigue siendo un mecanismo utilizado en EF para oprimir a los estudiantes homosexuales. Asimismo, estos comentarios homofóbicos tienden a condicionar la orientación sexual 'correcta', imponiendo un patrón esperado de conducta.

En relación con las actuaciones del profesorado, los estudios concuerdan en que no mencionan con frecuencia a personajes homosexuales como ejemplos y/o modelos. También señalan que los/as docentes casi nunca se enfrentan a los comportamientos heterosexistas $u$ homofóbicos y que no generan espacios seguros para las personas homosexuales (PIEDRA et al., 2013; SILVA; JAEGER; VALDIVIAMORAL, 2018). En general, el profesorado se muestra muy pasivo ante el acoso, toman pocas medidas y cuando lo hacen resultan ineficientes para superar las 
discriminaciones (BESERRA; BRITO; RIBEIRO, 2019; GILL et al., 2010; PIEDRA et al., 2013; PIEDRA et al., 2016; PRADO, 2017; PRADO; RIBEIRO, 2016; SILVA; JAEGER; VALDIVIA-MORAL, 2018). Sin embargo, una gran mayoría cree que se atienden estas situaciones en sus clases y crean espacios seguros (BESERRA; BRITO; RIBEIRO, 2019; PIEDRA et al., 2016). Esta discrepancia puede deberse a la gran dificultad que suelen tener los docentes para tratar asuntos relacionados con la sexualidad, en especial, la homofobia en las aulas (BESERRA; BRITO; RIBEIRO, 2019). Además, es posible que no se enfrenten a estas situaciones porque los puedan tildar de gays o lesbianas y poner en entredicho su aceptación social (SILVA; JAEGER; VALDIVIA-MORAL, 2018). Esto puede entenderse en un contexto lleno de resistencias para asumir la coeducación y valorar positivamente la visibilización de la homosexualidad en la escuela (PIEDRA; RAMÍREZ-MACÍAS; LATORRE, 2014).

Finalmente, la homonegatividad también forja sentimientos negativos entre las personas homosexuales al tener que mantener oculta su orientación sexual como estrategia para evitar la discriminación. Tal argumento lo describen las dos profesoras lesbianas del trabajo de Edwards, Brown y Smith (2016) cuando experimentan un nivel considerable de estrés y ansiedad al enfrentarse a una posible situación que les revele su identidad sexual o cuando temen ser juzgadas por las familias de sus estudiantes. Los participantes del estudio de Piedra et al. (2016) también indican que el profesorado y alumnado homosexual prefieren ocultar su orientación sexual real, minimizando la estigmatización de otros profesores y estudiantes. Otras estrategias de ocultación de la homosexualidad han consistido en mostrar una hiperfeminidad o hipermasculinidad exagerada para mantener un falso, pero aceptado estatus de heterosexual. Por ejemplo, una de las profesoras del estudio de Edwards, Brown y Smith, (2016) cuenta que intencionalmente 'coqueteó' con los hombres, cambió el nombre de las parejas femeninas a un equivalente masculino en sus conversaciones y también hacía cosas como dejarse el cabello largo para parecer más femenina. Estos resultados indican que, al menos en determinados contextos culturales, parecen existir altos niveles de homohisteria en el ámbito de la EF y el deporte, difiriendo de la disminución que apunta Anderson (2012) entre adolescentes del Reino Unido. En cierta forma, parece que el esquema panóptico, enunciado por Foucault (1999), está notablemente reflejado en la escuela y los homosexuales son neutralizados por la heteronormatividad, hasta el punto de que muchas personas no quieran identificarse explícitamente como homosexuales e incluso querer camuflarlo.

\subsection{PROPUESTAS PARA LAACCIÓN}

El análisis de los artículos de la revisión sistemática indica que todavía se está lejos de superar las actitudes, las creencias y los comportamientos homofóbicos y heterosexistas en la EF escolar, aun reconociendo homopositividad y mejoras en los últimos años. En estos artículos también se proponen diversas iniciativas y propuestas que emergieron de los estudios realizados para ayudar a disminuir y/o erradicar la homofobia de las clases de EF.

En primera instancia se detecta la necesidad de formar y sensibilizar al futuro profesorado en EF, así como dotarlo de herramientas de actuación adecuadas. En 
este sentido, las universidades juegan un papel fundamental como generadoras de cambio en el futuro profesorado, a través de la capacitación y la discusión académica en torno a la perspectiva de género y reconocimiento de grupos minoritarios (PIEDRA et al., 2013; PIEDRA; RAMÍREZ-MACÍAS; LATORRE, 2014; PRADO; RIBEIRO, 2016). Además, se deben propiciar cursos de coeducación y diversidad sexual a los actuales docentes de EF para que sean sensibles a estos temas y los incluyan en el desarrollo curricular de la materia (BESERRA; BRITO; RIBEIRO, 2019; GILL et al., 2010).

La segunda propuesta que mencionan algunos de los trabajos analizados consiste en aumentar el compromiso docente, ya que su rol es crucial para crear ambientes inclusivos y lograr que el alumnado se sienta seguro y cómodo en las clases (GILL et al., 2010; PIEDRA et al., 2016). El profesorado debe empoderarse como agente clave para lograr la emancipación e implicarse en contra de la homofobia y en pro del trato equitativo de todas las personas (EDWARDS; BROWN; SMITH, 2016; PIEDRA; RAMÍREZ-MACÍAS; LATORRE, 2014).

Como tercera propuesta, señalan la necesidad de seguir estudiando la homofobia en la EF escolar porque con el conocimiento generado se estará en disposición de iniciar propuestas de cambio, especialmente en contextos y lugares todavía exista poco conocimiento al respecto (PIEDRA et al., 2013; PIEDRA et al., 2016). Aquí resultan especialmente pertinentes los estudios cualitativos porque ofrecen conocimiento útil y profundo, así como los estudios de observación in situ para contrastar los hallazgos encontrados a través de cuestionarios de percepción u opinión (PIEDRA; RAMÍREZ-MACÍAS; LATORRE, 2014; PIEDRA et al., 2016; SILVA; JAEGER; VALDIVIA-MORAL, 2018).

La última propuesta consiste en dirigir la investigación a las experiencias, percepciones y comportamientos de las propias personas homosexuales, ya que la mayoría de las investigaciones disponibles se realizan con muestras de población general o heterosexuales y apenas se cuenta con unos pocos participantes con orientación sexual distinta a la dominante.

\section{CONCLUSIONES}

En este artículo de revisión se analizaron diez documentos correspondientes a estudios empíricos relacionados con la homofobia en la EF escolar y en el cual emergieron cuatro temas de interés: 1) la heteronormatividad y el heterosexismo imperante; 2) la existencia de homopositividad; 3) la homonegatividad atenuada; y 4) las propuestas para la acción.

Dentro de los hallazgos predominan las creencias y actitudes homofóbicas y heterosexistas en las clases de EF. Si bien los comportamientos homofóbicos no llegan a ser agresiones físicas, lo cual es muy positivo, se sigue señalando la existencia de insultos verbales o expresiones sutiles de violencia con bastante frecuencia. El heterosexismo y la homofobia se perciben frecuentemente en los estudios realizados con profesorado de EF y también en los estudios realizados con adultos y estudiantes universitarios cuando recuerdan la EF escolar. Aunque 
solo hemos analizado dos estudios realizados con escolares, se observa una gran disminución en las percepciones heterosexistas y homofóbicas entre los jóvenes, especialmente entre los escolares adolescentes del Reino Unido. Debido a que no existen investigaciones equivalentes en otros lugares, resulta difícil afirmar que esta tendencia vaya más allá del contexto cultural británico. Por ello, se requeriría estudiar especialmente estos temas con población más joven en otros contextos culturales.

La revisión también muestra que la influencia del deporte en el currículo escolar de EF está reproduciendo los valores heteronormativos, enfatizando la masculinidad tradicional y las actitudes conservadoras que resultan excluyentes, hostiles y sexistas. Por ello, es urgente que el profesorado de EF busque y se comprometa con iniciativas pedagógicas innovadoras e inclusivas en favor de la diversidad sexual y la coeducación, entendida como una educación en igualdad dentro de sus clases. Esta urgencia es más evidente ante la brecha existente entre lo que el profesorado cree que realiza y la percepción real por parte de los estudiantes en sus clases. Un desajuste similar se observa en el lenguaje utilizado en las clases, ya que en algunos estudios se recoge el uso del lenguaje inclusivo, pero en otros se refieren al uso frecuente de lenguaje peyorativo. Esto lamentablemente indica que la EF sigue siendo un espacio hostil y el profesorado y alumnado continúan perpetuando y reproduciendo la heteronormatividad. Como señala Gill et al., (2010) 'resulta sorprendente y perturbador que la educación física, siendo parte de un sistema educativo que debería ser un entorno seguro e inclusivo, sea percibido como un entorno hostil para las lesbianas y los gays, lleno de estereotipos y restricciones heterosexistas' (traducción nuestra) (p. 908). Este cuestionamiento deja en entredicho el papel de la escuela y la EF en particular, ya que no debería perjudicar, estigmatizar ni excluir a ciertos estudiantes cuya sexualidad no se corresponde con la sexualidad normativizada socialmente.

Finalmente, debemos señalar la necesidad de investigar la homofobia con muestras variadas y con diversidad de métodos cuantitativos y cualitativos porque todavía queda mucho por conocer, ya sea sobre variables o en contextos personales, sociales y ambientales diferentes.

\section{REFERENCIAS}

ANDERSON, Eric. Inclusive masculinity in a physical education setting. Thymos: Journal of Boyhood Studies, v. 6, n. 2, p. 151-165, 2012.

ANDERSON, Eric; BULLINGHAM, Rachael. Openly lesbian team sport athletes in an era of decreasing homohisteria. International Review for the Sociology of Sport, v. 50, n. 6, p. 647-660, 2015.

AYVAZO, Shiri; SUTHERLAND, Sue. Uncovering the secrets: Homophobia in physical education. Action in Teacher Education, v. 31, n. 3, p. 56-69, 2009.

BESERRA, José Thiago Soares; BRITO, Ahecio Kleber Araujo; RIBEIRO, Sergio Luis Galan. Homofobia nas aulas de educação física: um desafio para os professores de educação física do município de Buriti dos montes - Piaui. Form@re: Revista do Plano Nacional de Formação de Professores da Educação Básica, v. 7, n. 2, p. 81-90, jul./dez. 2019. 
BORRILLO, Daniel. Homofobia. Barcelona: Bellaterra, 2001.

CLARKE, Gill. Challenging heterosexism, homophobia and transphobia in physical education. In: STIDDER, Gary; HAYES, Sid. (eds.). Equity and Inclusion in Physical Education. London: Routledge, 2012. p. 87-101.

CLARKE, Gill. Queering the pitch and coming out to play: Lesbians in physical education and sport. Sport, Education and Society, v. 3, n. 2, p. 145-160, 1998.

CONNELL, Raewyn; PEARSE, Rebecca. Género Desde una Perspectiva Global. Traducción: GRAU, Arantxa; NAVAS, Almudena. Valencia: PUV, 2018.

CORNEJO, Juan. Bullying homofóbico en Chile: trayectoria histórica. Límite - Revista Interdisciplinaria de Filosofía y Psicología, v. 9, n. 30, p. 61-70, 2014.

COSTA, Angelo Brandelli; BANDEIRA, Denise Ruschel; NARDI, Henrique Caetano. Systematic review of instruments measuring homophobia and related constructs. Journal of Applied Social Psychology, v. 43, n. 6, p. 1324-1332, 2013.

CUNHA JUNIOR, Carlos Fernando F. da; MELO, Victor Andrade de. Homossexualidade, educação física e esporte: primeiras aproximações. Movimento, v. 3, n. 5, p. 18-24, 1996.

DEVÍS, José; FUENTES, Jorge; SPARKES, Andrew. ¿Qué permanece oculto del currículum oculto? Las identidades de género y de sexualidad en la educación física. Revista Iberoamericana de Educación, v. 39, p. 73-90, 2005.

DEVÍS-DEVÍS, Jose et al. Looking back into trans persons' experiences in heteronormative secondary physical education contexts. Physical Education and Sport Pedagogy, v. 23, n. 1, p. 103-116, 2018.

EDWARDS, Lisa L.; BROWN, David H.; SMITH, Lauren. 'We are getting there slowly': Lesbian teacher experiences in the post-section 28 environment. Sport, Education and Society, v. 21, n. 3, p. 299-318, 2016.

FERFOLJA, Tania. Lesbian teachers, harassment and the workplace. Teaching and Teacher Education, v. 26, p. 408-414, 2010.

FERREIRA, Ignacio; URRUTIA, Gerard; ALONSO-COELLO, Pablo. Revisiones sistemáticas y metaanálisis: bases conceptuales e interpretación. Revista Española de Cardiología, v. 64, n. 8, p. 688-696, 2011.

FOUCAULT, Michel. Vigilar y Castigar: Nacimiento de la prisión. México: Siglo XXI, 1999.

FRAÏSSÉ, Christéle; BARRIENTOS, Jaime. The concept of homophobia: A psychosocial perspective. Sexologies, v. 25, p.e65-e69, 2016.

GILL, Diane L. et al. Attitudes and sexual prejudice in sport and physical activity. Journal of Sport Management, v. 20, p. 554-564, 2006.

GILL, Diane L. et al. Perceived climate in physical activity settings. Journal of Homosexuality, v. 57, n. 7, p. 895-913, 2010.

GRANT, Maria J.; BOOTH, Andrew. A typology of reviews: an analysis of 14 review types and associated methodologies. Health Information and Libraries Journal, v. 26, p. 91-108, 2009.

GREENSPAN, Scott B.; GRIFFITH Catherine; WATSON, Ryan J. LGBTQ+ Youth's experiences and engagement in physical activity: A comprehensive content analysis.

Adolescent Research Review, v. 4, p. 169-185, 2019. 
GRIFFIN, Pat. Strong women, deep closets: Lesbians and homophobia in sport, Champaign: Human Kinetics, 1998.

HEMPHILL, Dennis; SYMONS, Caroline. Sexuality matters in physical education and sport studies. Quest, v. 61, n. 4, p. 397-417, 2009.

HEREK, Gregory M. Beyond "homophobia": A social psychological perspective on attitudes toward lesbians and gay men. Journal of Homosexuality, v. 10, n. 1-2, p. 1-21, 1984.

HEREK, Gregory M. Beyond 'homophobia': Thinking about sexual prejudice and stigma in the twenty-first century. Sexuality Research and Social Policy, v. 1, n. 2, p. 6-24, 2004.

HEREK, Gregory M.; MCLEMORE, Kevin A. Sexual Prejudice. Annual Review of Psychology, v. 64, p. 309-333, 2013.

HILL, Darryl B.; WILLOUGHBY, Brian L. The development and validation of the genderism and transphobia scale. Sex Roles, v. 53, n. 7/8, p. 531-544, oct. 2005.

HUDSON, Walter W.; RICKETTS, Wendell A. A strategy for the measurement of homophobia. Journal of Homosexuality, v. 5, n. 4, p. 357-372, 1980.

JAEGER, Angelita Alice et al. Professional formation in physical education: Homophobia, heterosexism and the possibilities of chances in student's perception. Movimento, v. 25, e25040, 2019.

JOY, Phillip; LARSSON, Håkan. Unspoken: exploring the constitution of masculinities in Swedish physical education classes through body movements. Physical Education and Sport Pedagogy, v. 24, n. 5, p. 491-505, 2019.

KOSCIW, Joseph G.; DIAZ, Elizabeth M. The 2005 National School Climate Survey: The experiences of lesbian, gay, bisexual and transgender youth in our nation's schools. New York: GLSEN, 2006. Disponible en: http://www.glsen.org . Acceso el: 12 mar. 2020.

LANDI, Dillon. Toward a queer inclusive physical education. Physical Education and Sport Pedagogy, v. 23, n. 1, p. 1-15, 2018.

LANDI, Dillon et al. LGBTQ Research in physical education: a rising tide? Physical Education and Sport Pedagogy, v. 25, n. 3, p. 259-273, 2020.

LARSSON, Håkan; REDELIUS, Karin; FAGRELL, Birgitta. Moving (in) the heterosexual matrix. On heteronormativity in secondary school physical education. Physical Education and Sport Pedagogy, v. 16, n. 1, p. 67-81, 2011.

LENSKYJ, Helen. Combating homophobia in sport and physical education. Sociology of Sport Journal, v. 8, p. 61-69, 1991.

LOGAN, Colleen R. Homophobia? No, Homoprejudice. Journal of Homosexuality, v. 31, n. 3, p. 31-53, 1996.

LONGARAY, Deisy Azevedo; RIBEIRO, Paulina Regina Costa; SILVA, Fabiane Ferreira da. 'Eu não suporto isso: Mulher com mulher e homem com homem': Analisando as narrativas de adolescentes sobre homofobia. Currículo sem Fronteiras, v. 11, n. 1, p. 252-280, jan./ jun. 2011.

MADUREIRA, Ana Flávia do Amaral; BARRETO, Ana Luiza Cruz Sá. Diversity, Social Identities, and Alterity: Deconstructing Prejudices in School. In: BRANCO. Angela Uchoa; LOPES-DE-OLIVEIRA, Maria Claudia (eds.). Alterity, Values, and Socialization, Cultural Psychology of Education. New York: Springer Publishing, 2018. p. 167-190. 
MADUREIRA, Ana Flávia do Amaral; BRANCO, Ângela Uchoa. Gênero, sexualidade e diversidade na escola a partir da perspectiva de professores/as. Temas em Psicologia, v. 23, n. 3, p. 577-591, 2015.

MADŽAREVIC, Goran. Measuring of homophobia: From traditional to modern instruments for prejudices toward gay people. Glasnik za društvene nauke, v. 8, p. 57-74, 2016.

MCCAUGHTRY, Nate et al. Sexuality Sensitive Schooling. Quest, v. 57, n. 4, p. 426-443, 2005.

MORENO, Alexander et al. Measuring homonegativity: Psychometric analysis of Herek's attitudes toward lesbians and gay men scale (ATLG) in Colombia, South America. Journal of Homosexuality, v. 62, n. 7, p. 924-935, 2015.

MORROW, Ronald G.; GILL, Diane L. Perceptions of homophobia and heterosexism in physical education. Research Quarterly for Exercise and Sport, v. 74, n. 2, p. 205-214, 2003:

MOSCOSO, David; PIEDRA Joaquín . El colectivo LGTBI en el deporte como objeto de investigación sociológica. Estado de la cuestión. Revista Española de Sociología, v. 28, n. 3 , p. 501-516, 2019.

NICOLINO, Aline Silva; PARAISO, Marlucy Alves. Escolarização da Sexualidade: O silencio como pratica pedagógica da educação física. Movimento, v. 24, n. 1, p. 93-106, jan./mar. 2018

PEREIRA, Erik Giuseppe Barbosa et al. Os estudos de gênero e masculinidade e seus reflexos para a educação física. Revista Brasileira de Ciência e Movimento, v. 23, n. 1, p. 146-156, 2015.

PICHARDO, José Ignacio. El estigma hacia personas lesbianas, gays, transexuales y bisexuales (LGBT). En: GAVIRIA, Elena; GARCÍA-AEL, Cristina; MOLERO, Fernando (coord.). Investigación-acción: aportaciones de la investigación a la reducción del estigma. Madrid: Sanz y Torres, 2012. p. 111-125.

PICHARDO, José Ignacio. Homofobia y acoso escolar. En: PICHARDO, José Ignacio et al. (eds.). Adolescentes ante la diversidad sexual: Homofobia en los centros educativos. Madrid: Catarata, 2009. p. 19-35.

PIEDRA, Joaquín et al. Analysis of homophobia during Physical Education lessons in Spain. In: TODARO Ricky (ed.). Handbook of Physical Education Research. New York: Nova Science Publishers, Inc, 2014. p. 143-164

PIEDRA, Joaquín et al. Homophobia and heterosexism: Spanish physical education teachers' perceptions. Sport in Society, v. 19, n. 8-9, p. 1156-1170, 2016.

PIEDRA, Joaquín et al. Homofobia, heterosexismo y educación física: percepciones del alumnado. Profesorado. Revista de Currículum y Formación de Profesorado, v. 17, n. 1, p. 325-338, 2013.

PIEDRA, Joaquín; GARCÍA-PÉREZ, Rafael; CHANNON, Alexander G. Between homohysteria and inclusivity: Tolerance towards sexual diversity in sport. Sexuality \& Culture, v. 21, p. 1018-1039, 2017.

PIEDRA, Joaquín; RAMÍREZ-MACÍAS, Gonzalo; LATORRE, Agueda. Visibilizando lo invisible: creencias del profesorado de educación física sobre homofobia y masculinidades. Retos: Nuevas Tendencias en Educación Física, Deporte y Recreación, v. 25, p. 36-42, 2014.

PRADO, Vagner Matias do. Entre queerpos e discursos: Normalização de condutas, homossexualidades e homofobia nas práticas escolares da educação física. Práxis Educativa, v.12, n. 2, p. 501-519, maio/ago. 2017. 
PRADO, Vagner Matias do; RIBEIRO, Arilda Ines Miranda. Escola, homossexualidades e homofobia: rememorando experiências na educação física escolar. Revista Reflexão e Ação, v. 24, n. 1, p. 97-114, jan./abr. 2016.

PRADO, Vagner Matias do; RIBEIRO, Arilda Ines Miranda. Homofobia y educación sexual en la escuela Percepciones de homosexuales en enseñanza secundaria. Revista Retratos de la Escuela, v.9, n. 16, p. 137-152, 2015.

RAMÍREZ-DÍAZ, Alejandro; CABEZA-RUIZ, Ruth. Actitudes hacia la diversidad sexual en el deporte en estudiantes de educación secundaria. Retos, v. 38, p. 654-660, 2020.

RELATIONSHIPS education, relationship and sex education (RSE) and health education. [United Kingdom]: Department for Education, 25 jun. 2019. Disponible en: https://www.gov. uk/government/publications/relationships-education-relationships-and-sex-education-rseand-health-education. Acceso el: 4 May 2020.

SCHARAGRODSKY, Pablo Ariel. Entre machos y no tan machos: El caso de la educación física escolar argentina. Breve genealogía de la educación física escolar argentina o acerca de cómo construir masculinidad y femineidad. Apunts Educación Física y Deportes, v. 72, p. $41-48,2003$.

SEARS, James T.; WILLIAMS, Walter L. Overcoming heterosexism and homophobia. New York: Columbia University, 1997.

SILVA, Paula; JAEGER, Angelita Alice; VALDIVIA-MORAL, Pedro. Percepción de los estudiantes sobre comportamientos homofóbicos y heterosexistas en educación física.

Revista de Psicología del Deporte, v. 27, n. 2, p. 39-46, 2018.

SQUIRES, Sarah L.; SPARKES, Andrew C. Circles of silence: Sexual identity in physical education and sport. Sport, Education and Society, v. 1, n. 1, p.77-101, 1996.

SYKES, Heather; GOLDSTEIN, Tara. From performed to performing ethnography: translating life history research into anti-homophobia curriculum for a teacher education program. Teaching Education, v. 15, n. 1, p. 41-61, mar. 2004.

TENA, Olivia. Análisis ético de la homofobia. En: MUÑOZ, Julio (coord.) Homofobia: Laberinto de la ignorancia. México: UNAM, Centro de Investigaciones Interdisciplinarias en Ciencias y Humanidades, 2012. p. 91-108.

ZAWACKI-RITCHER, Olaf et al. Systematic Reviews in Educational Research: Methodology, Perspectives and Application. Wiesbaden: Springer VS, 2020. 
Resumo: A Educação Física (EF) é uma das disciplinas escolares que mais promove um ambiente hostil e marginal para os homossexuais. Este trabalho visa analisar artigos empíricos sobre crenças, atitudes e comportamentos homofóbicos presentes na EF Escolar por meio de uma revisão sistemática. Além de vários aspectos bibliométricos de interesse, quatro grandes temas emergiram da análise: 1) heteronormatividade e heterossexualismo predominante; 2) existência de homopositividade; 3) homonegatividade atenuada; e 4) propostas de ação. Essas questões são discutidas em detalhes e conclui-se que, apesar das melhorias, a homofobia ainda é generalizada. Além disso, há a necessidade de investigar a homofobia com amostras variadas e uma diversidade de métodos porque ainda há muito a aprender, seja sobre variáveis ou em diferentes contextos pessoais, sociais e ambientais.

Palavras chave: Homofobia. Normas de gênero. Revisão sistemática. Educação Física.

\begin{abstract}
As a school subject, Physical Education (PE) is highly fertile for hostile and marginal environments for homosexuals. Therefore, this paper aims to analyse empirical articles on homophobic beliefs, attitudes and behaviours present in school PE by means of a systematic review. In addition to several bibliometric aspects of interest, four major themes emerged from the analysis: 1) dominant heteronormativity and heterosexism; 2) the existence of homopositivity; 3) attenuated homonegativity; and 4) proposals for action. These issues are discussed in detail and lead to the conclusion that while there have been improvements, homophobia is still widespread. In addition, homophobia has to be investigated using various examples and diverse methods because there is still much to be learned, both about variables and in different personal, social and environmental contexts.
\end{abstract}

Keywords: Homophobia. Gender norms. Systematic review. Physical education. 


\section{LICENCIA DE USO}

Este es un artículo publicado en Open Access bajo la licencia Creative Commons Atribución 4.0 Internacional (CC BY 4.0), que permite su uso, distribución y reproducción en cualquier medio, siempre que la obra original esté correctamente citada. Más información en: http://creativecommons.org/licenses/by-nc/4.0

\section{CONFLICTO DE INTERESES}

Los autores han declarado que no existe conflicto de intereses en este trabajo.

\section{CONTRIBUCIONES DE LOS AUTORES}

Angélica María Sáenz Macana: Diseño del estudio, elaboración de la estrategia de búsqueda, búsqueda de documentos, análisis de documentos, redacción inicial del manuscrito, redacción final del manuscrito.

José Devís Devís: Diseño del estudio, elaboración de la estrategia de búsqueda, análisis de los documentos, revisión de la redacción inicial del manuscrito, redacción final del manuscrito.

\section{FINANCIACIÓN}

El presente trabajo se llevó a cabo sin ningún apoyo financiero.

\section{CÓMO HACER REFERENCIA}

SÁENZ-MACANA, Angélica María; DEVIS-DEVÍS, José. La homofobia en la educación física escolar: una revisión sistemática. Movimento, v.26, p. e26072, ene./dic. 2020. Disponible en: https://seer.ufrgs.br/Movimento/article/view/104750. Acceso en: 19 set. 2020. DOI: https://doi.org/10.22456/1982-8918.104750

\section{RESPONSABILIDAD EDITORIAL}

Alex Branco Fraga*, Elisandro Schultz Wittizorecki*, Ivone Job*, Mauro Myskiw*, Raquel da Silveira*

*Universidad Federal de Rio Grande do Sul, Escuela de Educación Física, Fisioterapia y Danza, Porto Alegre, RS, Brasil 